\title{
REDUCTION OF EXPERIMENTALLY INDUCED NEURONAL DEATH IN SPINAL GANGLIA OF THE CHICK EMBRYO BY NERVE GROWTH FACTOR ${ }^{1}$
}

\author{
VIKTOR HAMBURGER* AND JOSEPH W. YIP $\ddagger^{2}$ \\ *Department of Biology, Washington University, St. Louis, Missouri 63130 and $\ddagger$ Department of Physiology, University of \\ Pittsburgh School of Medicine, Pittsburgh, Pennsylvania 15261
}

Received June 10, 1983; Revised September 21, 1983; Accepted September 21, 1983

\begin{abstract}
Extirpation of the wing bud in 2-day chick embryos results in a conspicuous degeneration of neurons in both populations of brachial dorsal root ganglia (DRG). Daily injections of 1 to $6 \mu \mathrm{g}$ of nerve growth factor (NGF), beginning at $4 \frac{1 / 2}{2}$ days of incubation, rescued all small, late differentiating (DM) neurons and approximately $50 \%$ of large, early differentiating (VL) neurons, which would have died otherwise. The fact that NGF is an effective substitute for the hypothetical trophic maintenance factor for DRG which is normally produced by limb tissues strengthens our belief that NGF is identical with this factor.

The control experiment, i.e., wing extirpation without NGF injections, revealed an inconsistency with previous data. Experiments on a number of different neuronal units had shown rather consistently that the period of experimentally induced neuron degeneration, caused by removal of the target, is synchronous with the period of normally occurring neuronal death in the same neuronal unit. This synchrony rule is violated by the VL population of brachial DRG. In this unit, the peak of degeneration resulting from wing bud extirpation occurs considerably earlier than the peak of normally occurring neuronal death. The competition hypothesis for the explanation of neuronal death had been based, in part, on the synchrony rule. We discuss the question of whether the deviation from the synchrony rule observed in our material represents a serious challenge to the competition hypothesis.
\end{abstract}

Nerve growth factor (NFG) is known to be a trophic maintenance factor for embryonic dorsal root ganglia (DRG) and sympathetic neurons (Levi-Montalcini and Angeletti, 1968). It is assumed that NGF is produced by the target structures of these neurons, but the available assays are not sufficiently sensitive to provide clear evidence for the presence of NGF in these tissues (Harper and Thoenen, 1980). In a previous publication (Hamburger et al., 1981), we obtained indirect support for the notion that NGF is the naturally occurring maintenance factor for DRG of the chick embryo. We found that daily injections of NGF, administered from $4 \frac{1}{2}$ days on, re-

\footnotetext{
${ }^{1}$ This work was supported by Grant NS05731 of the National Institute of Neurological and Communicative Disorders and Stroke, National Institutes of Health and an intramural grant from the University of Pittsburgh. We are grateful to Dr. Eugene Johnson of the Department of Pharmacology, Washington University School of Medicine, for the generous supply of nerve growth factor. We thank Mrs. Helen Gregory for excellent technical assistance and Mrs. Irma Morose for typing the manuscript.

${ }^{2}$ To whom correspondence should be addressed.
}

sulted in a substantial reduction of normally occurring neuronal death in the VL population of thoracic DRG and in the rescue of practically all sensory neurons in the VL population of brachial ganglia and the DM populations of both brachial and thoracic ganglia which would have died otherwise. (VL refers to large early differentiating neurons in a ventrolateral position and DM to small, late differentiating neurons in a dorsomedial position.) In the present investigation we have subjected the assumption that NGF is identical with, or closely related to, the maintenance factor normally produced by limb tissues to a more severe test. The extirpation of early limb buds is known to increase neuron degeneration very substantially both in brachial DRG (Hamburger and Levi-Montalcini, 1949) and in the lateral motor columns (Hamburger, 1934, 1958). By combining early wing bud extirpation with daily injections of NGF we were able to rescue nearly all brachial DM neurons and a substantial number of brachial VL neurons.

Unexpectedly, the control series, that is, wing bud extirpation without NGF injections, revealed a surprising 
phenomenon which had been overlooked before and which compels us to re-evaluate some widely held notions concerning neuronal death. All studies of both naturally occurring and experimentally induced death that have been done so far have confirmed the observation (Hamburger and Levi-Montalcini, 1949) that both events occur synchronously. The synchrony, combined with the observation that neuronal death occurred at the time when the axons arrived at the target area led to the competition hypothesis. It states that naturally occurring neuronal death results from unsuccessful competition for an inadequate supply of a target-produced trophic maintenance factor and that experimentally induced neuronal death represents a quantitative enhancement of naturally occurring death. We have now found that in the brachial VL population the peak of degeneration resulting from wing bud extirpation occurs considerably earlier than the peak of natural neuronal death. This is a clear violation of the synchrony rule. The question of whether this finding constitutes a challenge to the competition hypothesis will be taken up under "Discussion."

\section{Materials and Methods}

Chick embryos (White Leghorn obtained from Sacks Farm, Evans City, PA) were incubated in a forced-draft incubator at $37^{\circ} \mathrm{C}$ and $70 \%$ relative humidity.

Wing bud extirpations (WX series). The operations were done at stages 17 to 19 (21/2 to 3 days; Hamburger and Hamilton, 1951). A window was made in the shell using a rotary sander. The right wing bud was exposed by cutting the amnion with iridectomy scissors. Using a glass needle, a small hole was made in the body wall above the wing bud. The wing bud and the immediately adjacent somatopleure were then removed by inserting a pair of iridectomy scissors into the hole and cutting longitudinally between the base of the wing bud and the edge of the somites and transversely in front and behind the wing bud. The window was then sealed with a coverslip and paraffin. The embryos showed later an ectopia viscerum with heart and sometimes part of the viscera protruding. However, the formation of the axial organs, DRG, and sympathetic ganglia and viscera was normal.

NGF injections (NGFX series). Purified NGF was dissolved in $0.03 \%$ acetic acid and diluted with phosphatebuffered saline containing bovine serum albumin (BSA). The final solution, which contained $0.5 \mathrm{mg} / \mathrm{ml}$ of NGF and $6 \mathrm{mg} / \mathrm{ml}$ of BSA, was filtered with a Millipore filter $(0.22 \mu \mathrm{m}$ pore size $)$ before use. Beginning at $3 \frac{1}{2}$ days (stage 20), daily injections of NGF were made with a Hamilton syringe into the amniotic sac of the embryo. Increasing doses of NGF were used to match the increase in size of the embryo (third day, $1 \mu \mathrm{g}$; fourth and fifth days, $2 \mu \mathrm{g}$; sixth to eleventh days, $6 \mu \mathrm{g}$ ). In four embryos, massive doses of NGF (six times the normal dose) were injected into the yolk sac to examine the dose effect of NGF on the second peak of degeneration on VL neurons (see below).

Histological preparation. The embryos were fixed with Carnoy solution, embedded in paraffin, and sectioned transversely at $10 \mu \mathrm{m}$. Serial sections were mounted and stained with thionine or Pappenheim's solution for histological examinations.
Identification of $D R G$. To identify ganglia, a reconstruction of the DRG adjacent to segments 12 to 18 of the spinal cord was made. The lengths of all ganglia on both sides, along the rostrocaudal axis, were plotted on graph paper, the ganglia were drawn, assuming an arbitrary width; the emergence of the segmental nerves and their convergence in the brachial plexus were traced, as well as the proximal parts of the brachialis and radialis nerves. DRG 13 to 16 , which contribute to the plexus, were thus identified.

Identification and plotting of degenerating neurons. This method was described in a previous publication (Hamburger et al., 1981). Briefly, the curves representing the numbers of degenerating cells for the VL and DM populations (Figs. 1 and 2) were obtained by counting all degenerating cells in alternate sections, along the length of the ganglion, on both sides, and separately for VL and DM populations. The totals were multiplied by two. Counts were made for both ganglia 15 and 16 . The left sides served as controls in both WX and NGFX series.

Degenerating cells were identified under oil immersion at a magnification of $\times 750$. They appear in a variety of forms, ranging from pyknotic nuclei in a seemingly normal perikaryon, to condensed, deeply stained spheres or vacuolated spheres. Macrophages containing debris were rare.

The criteria to distinguish VL and DM cells were: topographic position (ventrolateral versus dorsomedial) and, more importantly, the size of the neurons immediately surrounding a degenerating cell. Some arbitrariness in the decision is unavoidable, partly because the line of demarcation between the two populations becomes less distinct, due to the penetration of strands of VL cells into the DM territory, and partly because neuron sizes become modified-NGF treatment results in their enlargement and wing extirpation in cell atrophy, preceding death. Hence some neurons of intermediate size are particularly difficult to identify. However, the consistency of the data, indicated in the relatively low standard errors, indicates that errors in one or the other direction cancel each other out. The important early stages, 25 to 30 , are not seriously affected by this source of error. Very small dark spheres, which are probably degenerating glia, were not counted.

In the WX series, six to nine embryos were used for each stage, from 24 to 34 ( $4 \frac{1}{2}$ to 8 days), four to five embryos were used for the oldest stages. In the NGFX series, five to nine embryos were used for each stage. The critical point for the peak of VL degeneration in the NGFX series is documented by 10 ganglia. Counts were made for ganglia 15 and 16 at all stages. We consider the fact that the curves for both populations and for both series WX and NGFX are nearly identical in both ganglia as further indication of the reliability of our counting procedure. All experiments and injections were made by J. W. Y. and all counts by V. H.

\section{Results}

Since we found no differences in the data for DRG 15 and 16, we represent only the results obtained for DRG 15.

VL population controls (Fig. 1, open circles, solid lines; 


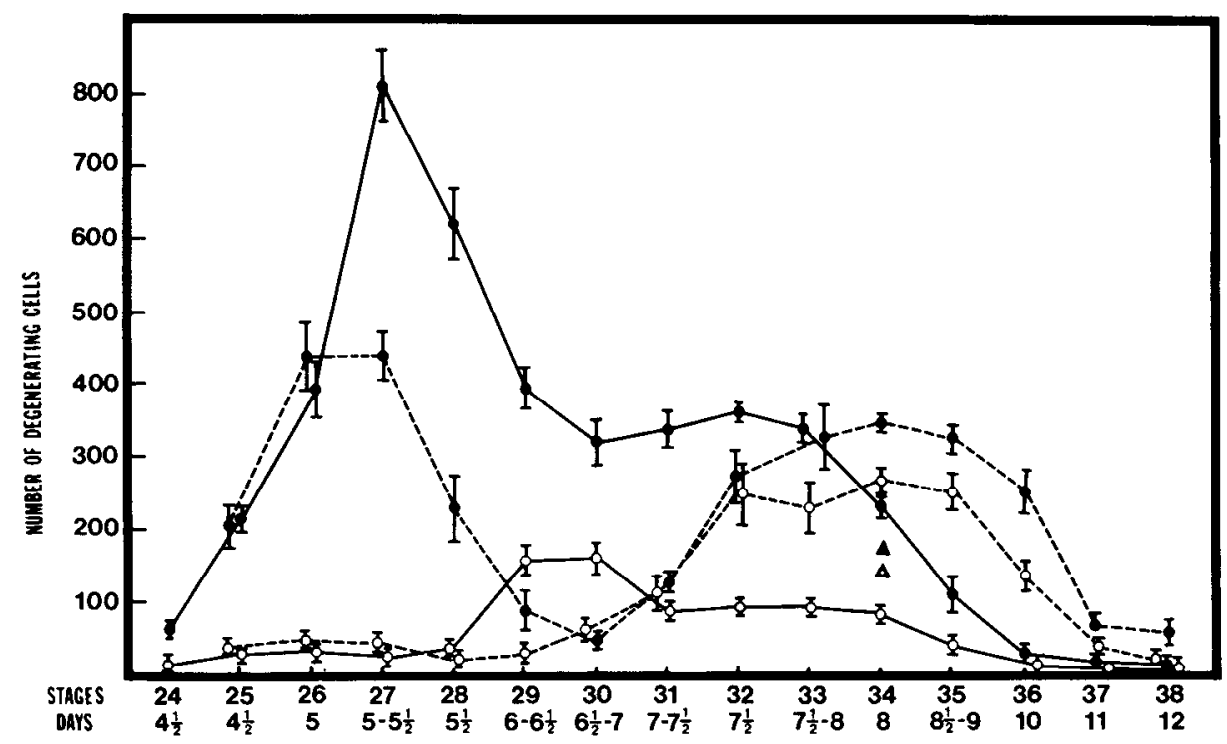

Figure 1. Degeneration in the VL population of brachial ganglion 15.

Figures 1 to 3 . Figures 1 to 3 show the numbers of degenerating neurons with the standard error of the mean. Each point represents the average of 6 to 10 ganglia. $\longrightarrow$, wing bud extirpation; $\mathrm{O}-\mathrm{O}$, normally occurring neuronal death; -- - combination of wing bud extirpation and NGF injections; $\mathrm{O}---\mathrm{O}$ controls, NGF injections only; $\boldsymbol{\Delta}$, wing bud extirpation combined with injection of heavy dose $\mathrm{NGF}, \triangle$, controls, heavy dose NGF injections only (see the text).

open circles, dashed lines). We have compared the left (control) ganglia of the WX series with previously published data for normal DRG 15, and the left (control) ganglia of the NGF injection series with previously obtained data for degeneration in NGF-injected embryos (compare Fig. 1 with Fig. 6 in Hamburger et al., 1981). Both sets of data are in good agreement. The normal degeneration period in brachial ganglia is much later than that in thoracic ganglia, and the amount of degeneration is low, compared to that in the brachial DM population and in both VL and DM of thoracic ganglia. Furthermore, daily NGF injections result in the survival of most VL neurons. However, it had been observed previously that some of the VL cells which had been rescued between 6 and $7 \frac{1}{2}$ days died later; a second, not very conspicuous peak of degeneration occurred at 8 to 9 days. The present material showed the same phenomenon; however, the secondary loss of cells was considerably greater than in the material presented in 1981. We shall take up this point in the discussion.

$V L$ degeneration following wing bud extirpation (WX) (Fig. 1, solid circles, solid lines). As was shown earlier (Hamburger and Levi-Montalcini, 1949), target deprivation results in a dramatic increase in the number of degenerating VL neurons. The new, quantitative data show that, at its peak, the average number is 800 , as compared to 150 in normal brachial DRG. However, what was unexpected is a conspicuous shift of the degeneration period to considerably earlier stages than in normal embryos. Whereas in normal DRG 15 the degeneration period begins between stages 28 and 29 (6 to $6 \frac{1}{2}$ days), it begins in target-deprived ganglia already between stages 24 and 25 ( $4^{1 / 2}$ days). The peak shifts from stages 29 to $30\left(6^{1 / 2}\right.$ to 7 days) to stage $27\left(5 \frac{1}{2}\right.$ days). Apart from this difference and the much higher amount of neuronal death in experimental ganglia, we find a further difference in the shape of the curves. In normal ganglia, a low peak extends from stage 29 to $30\left(6^{1 / 2}\right.$ to 7 days), followed by a gradual decline. In target-deprived ganglia, there is a steep rise to a sharp peak at stage 27 (51/2 days) followed by a steep decline to stage $29\left(6^{1 / 2}\right.$ days). Subsequently, there is a plateau and a final phase of gradual decline to the base line, which is reached at stage 36 . The main characteristic of the change of response in target-deprived ganglia seems to be a pronounced acceleration of the degeneration process. However, at close inspection one finds that the general trend of both curves is the same; a peak period followed by a plateau and finally a gradual decline to the base line.

$V L$ degeneration following a combination of wing bud extirpation and NGF injections. (NGFX, Fig. 1, solid circles, dashed lines). No effects were noted up to stage 26 (5 days); that is, the first two injections were ineffective. One-half day later, when degeneration reaches its peak in the WX ganglia, degeneration is reduced by $46 \%$. The subsequent sharp decline in the case of wing bud extirpation is paralleled in the NGFX series, at a much lower level. Neuronal degeneration at stages 28,29 , and 30 is reduced by $63 \%, 78 \%$, and $86 \%$, respectively. In other words, the NGF treatment rescues a very considerable number of neurons. However, this effect is only temporary. After stage 30 ( 7 days), a second degeneration period begins which extends over 4 days and reaches peak values of over 300 degenerating cells per stage over a prolonged period. In the absence of data for total population size at different stages, it is not possible to determine whether all neurons that were rescued earlier die or whether some survive during this second degeneration period.

DM population controls (Fig. 2, open circles, solid lines). 


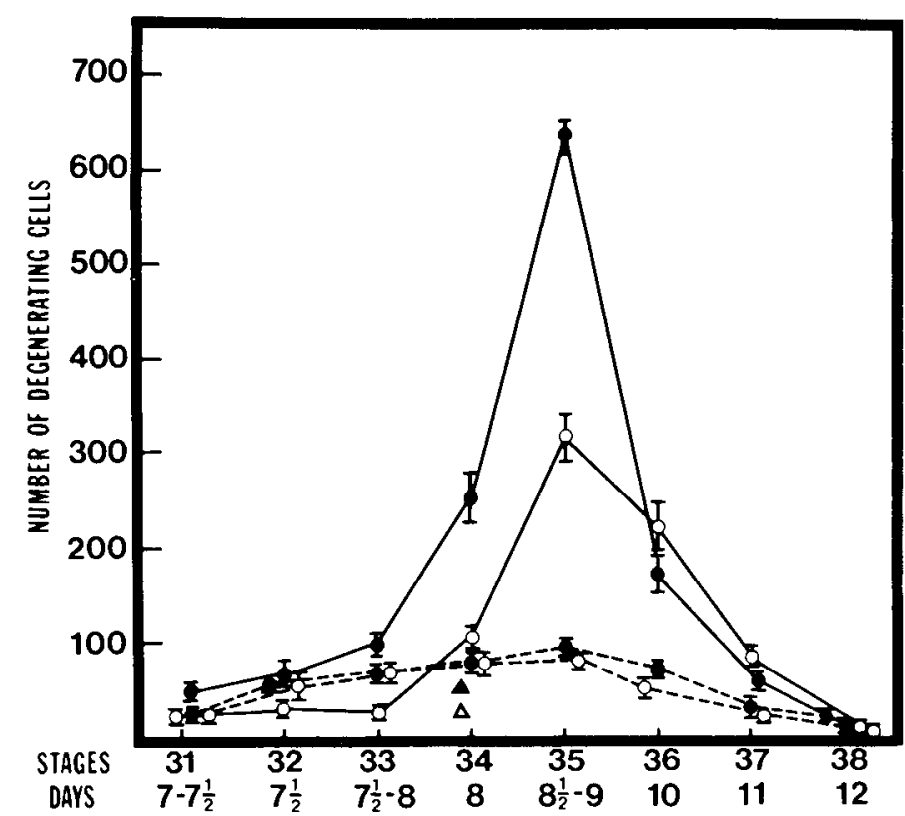

Figure 2. Degeneration in the DM population of brachial ganglion 15.

The present data for neuron loss in the DM population of normal brachial ganglia resemble closely those obtained in the previous study (compare Fig. 2 with Fig. 7 in Hamburger et al., 1981). The only difference is that the peak value at stage 35 (9 days) is somewhat above 300 degenerating cells, compared to 400 in the previous investigation. This holds for ganglia 15 and 16 . We have no explanation for this difference. A comparison of the control ganglia in the NGFX series with corresponding ganglia in normal NGF-injected embryos (Hamburger et al., 1981) shows good agreement; the NGF treatment reduces neuronal death in the DM population almost to the base level.

$D M$ degeneration following wing bud extirpation (WX, Fig. 2, solid circles, solid lines). The cell loss which is observed in the DM population of normal brachial ganglia is doubled at the peak period if the target is removed. This increase seems to be much less impressive than that reported above for the VL population. However, one has to take into account that in normal brachial ganglia the neuronal loss in the VL population is insignificant compared to that in the DM population. The most interesting point is that the degeneration periods in normal and target-deprived ganglia are synchronous, in contrast to the shift to an earlier stage which was described above for the VL population of target-deprived ganglia.

$D M$ degeneration following a combination of wing bud extirpation and NGF injections (NGFX, Fig. 2, solid circles, dashed lines). As a result of the NGF treatment, nearly all of the DM neurons which would have died, following wing bud extirpation, did survive. They are all alive at stage 38 (12 days) which is the end point of our observations. There is no indication of a second degeneration period, as was found in the VL population.

Injection of heavy doses of NGF (Fig. 1, solid triangle). Four embryos with wing bud extirpations received daily doses six times the dose administered to the other NGFX cases. The rationale for this test was that, if the second degeneration period in the VL neurons is due to an inadequate supply of NGF, a massive increase in the dose should reduce or abolish the second period. The embryos were sacrificed at stage 34 , that is, at the peak of the second degeneration period. Indeed, the higher dose of NGF reduces the number of degenerating neurons, although it does not rescue all VL neurons. Whereas the average number of degenerating VL cells is 345 for embryos which had received the regular dose ( $n$ $=6$ ), the average for the embryos, which had received the heavy dose $(n=4)$, is 170 ; thus, the amount of degeneration is cut in half.

\section{Discussion}

A re-evaluation of the effects of target removal on the $V L$ population of DRG. Before we discuss the central theme of this investigation, we shall deal with a novel aspect of the effects of wing bud extirpation on brachial DRG. The new data reveal that in the experimental situation the degeneration period of the brachial VL population occurs at a much earlier stage than in normal brachial DRG. The peak of degeneration (identified by counts of degenerating neurons) occurs in normal brachial DRG at stages 29 to 30 (6 to 7 days), but in targetdeprived DRG it is shifted to a much earlier stage (27,5 days). The initial phase of massive degeneration in the target-deprived VL population occurs even before the onset of degeneration in normal ganglia (Fig. 1). In the later phase, from stage 31 on, the curves for normal and target-deprived VL neurons run parallel, although, of course, the absolute numbers of degenerating cells are much larger in experimental than in normal embryos. One can state that target removal has accelerated the degeneration process considerably.

This shift seems to violate a basic principle enunciated first in the original report on degeneration of DRG neurons (Hamburger and Levi-Montalcini, 1949). It was established then that normally occurring death (in thoracic VL neurons) and experimentally induced neuronal death (in brachial VL neurons) occur synchronously and approximately at stages when the axons arrive at the target area. This observed synchrony, in turn, led to the competition hypothesis which postulates that all axons from a neuronal unit reach the target area at about the same time and compete there for a limited supply of a maintenance factor. The dying neurons would be those whose axons had lost out in the competition for the trophic agent. The general acceptance of the competition hypothesis is based largely on the fact that practically all later investigations of the phenomenon of neuronal death confirmed the synchrony of normally occurring and experimentally induced death. For instance, it was found in the lateral motor column of the chick (Hamburger, 1975), in the trochlear nucleus (Cowan and Wenger, 1967), and in the ciliary ganglion (Landmesser and Pilar, 1974). We shall refer to this as the synchrony rule (general review in Hamburger and Oppenheim, 1982).

Obviously, the present case is an exception to this rule. Does it represent a serious challenge to the competition hypothesis? Fortunately, an explanation can be offered which actually strengthens the argument for the com- 
petition hypothesis, rather than weakening it. In a recent re-investigation of neuronal death in DRG of the chick embryo (Hamburger et al., 1981) data were obtained for both brachial and thoracic DRG which extend and, in some respects, correct the observations presented in the previous publication (Hamburger and Levi-Montalcini, 1949) and are relevant for the present situation. We had originally disregarded the seemingly negligible degeneration of brachial VL neurons in normal ganglia, but the re-investigation has established a distinct, although not very conspicuous, degeneration period for the brachial VL population. But the most striking new finding in Hamburger et al. (1981) concerns the early massive degeneration of VL neurons in normal thoracic ganglia. It was shown that many of them die when their axons have barely reached the dermis; others die even earlier, before axon outgrowth. We know this because the EM studies of Pannese (1976) have shown that most of the cells which die between 4 and $6 \frac{1}{2}$ days, that is, during the peak phase of VL death in thoracic ganglia, are still in the bipolar stage or intermediate between the bipolar and pseudo-unipolar stage. Furthermore, Carr and Simpson (1982) have shown autoradiographically that $14 \%$ of the neurons which die during the peak period ( $5^{1 / 2}$ days) are labeled: this implies that some neurons or neuron precursors die while still engaged in proliferation. These data were interpreted in terms of either a very short supply of a maintenance factor produced by tissues adjacent to the ganglia, or a very early dependency of VL neurons on an extraneous supply of a trophic agent, or both.

These findings concerning VL cells in thoracic ganglia have a direct bearing on the present case. We suggest that wing bud extirpation, by removing the major source of the requisite trophic maintenance factor, reduces its supply to an amount equivalent to that available at the thoracic level: hence the target-deprived brachial VL neurons are in the same predicament as the normal thoracic neurons, and they respond accordingly. In this respect, the target-deprived brachial ganglia have been transformed into the equivalent of normal thoracic ganglia.

This interpretation is borne out by the remarkable fact that the curve for VL degeneration in target-deprived brachial ganglia is almost an exact replica of that for VL neurons in normal thoracic ganglia (Fig. 3). In both instances, a steep rise begins at stage 24 ( $4^{1 / 2}$ days); the peak at stage $27\left(5 \frac{1}{2}\right.$ days $)$ is followed by an equally steep decline to stage 29 ( $6^{1 / 2}$ days). Subsequently, a plateau is maintained for several days, followed by a gradual decline to the base which is reached at stages 34 and 35 , respectively ( 8 to 9 days). It should be pointed out that the absolute numbers of degenerating cells are higher in the experimental cases. This can be explained by the fact that part of the brachial flank tissue was removed with the wing bud, and the wound had not healed over.

In summary, the "shift" of the degeneration period in the VL neurons of target-deprived brachial ganglia to earlier stages, which represents an exception to the synchrony rule and, therefore, a challenge to the competition hypothesis, is, in fact, exactly what would have been predicted by this hypothesis in its most general formulation, that is, with emphasis on competition for a restricted supply of a trophic maintenance agent. It seems now that the synchrony rule is valid for those instances in which all axons had reached their target before the onset of naturally occurring death, and that all previously studied cases, with the single exception of the VL neurons in the DRG of the chick embryo, belong to this category. As a corollary, the case of the VL neurons demonstrates clearly that the observance of the synchrony rule is not a necessary prerequisite for the validation of the competition hypothesis.

But this interpretation still leaves a significant point unresolved. It is clear from earlier observations on normal thoracic DRG and the present observations on target-deprived brachial DRG that at least part of the VL

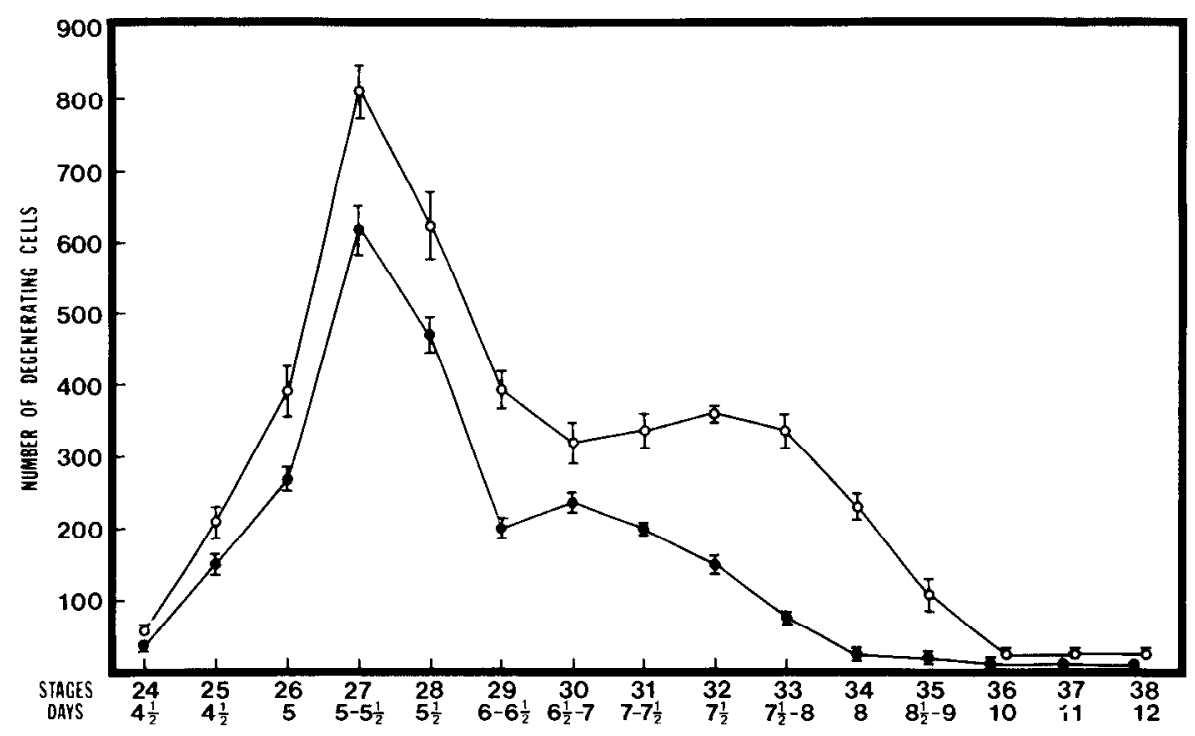

Figure 3. Comparison of VL degeneration in brachial ganglion 15, following wing bud extirpation (upper curve) and normally occurring neuronal death in thoracic ganglion 18 (lower curve, the latter from Figure 4 in Hamburger et al., 1981). 
population is highly vulnerable to inadequate supply of the requisite trophic agent, at a very early stage of its differentiation. We have suggested that in the brachial ganglia this requirement is met by an adequate supply produced by limb tissues. But why does the brachial VL population show in normal development a distinct degeneration period 2 days later, when the limb has grown considerably and one would expect no shortage of the agent? A possible explanation would be that we are dealing with two subpopulations with different trophic requirements that become acute at different stages. A heterogeneity of the VL population had been suggested before (Hamburger et al., 1981, p. 67) and has actually been demonstrated by Honig (1982). If the assumption of two subpopulations of VL cells is correct, and if we are actually dealing with two separate degeneration periods, that is, an early one with a peak at stage 27 and a later one with a peak at stages 29 and 30 , then it would be misleading to speak of a shift, or acceleration, of the degeneration period, as we did above. One can speak of a shift only if one deals with a single population.

Paradoxically, the original statement of the synchrony rule (Hamburger and Levi-Montalcini, 1949) was based on data which now turn out to be the only exception to this rule detected so far. Since, as was mentioned, we had discounted the moderate amount of VL neuron death which actually occurs in normal brachial ganglia, we had compared the experimentally induced degeneration in brachial DRG with normally occurring death in thoracic $\mathrm{DRG}$, and since they are synchronous-as is now documented in detail - we generalized from this comparison. This is not the first time that an inappropriate interpretation of data has led to a valid paradigm.

An acceleration of neuronal death caused by target deprivation has been reported for the lumbar lateral motor column of the chick embryo (Oppenheim et al., 1978). However, the acceleration is much less pronounced than in the brachial DRG.

Neuronal death in the DM population. In this instance, the synchrony rule is upheld; the pattern of degeneration is the same in normal and target-deprived brachial DRG. A sharp rise beginning at stage $33(71 / 2$ days) reaches a peak at stage 35 (9 days), which is followed by an equally sharp decline. The base line is reached in both instances at stage 38 (12 days) (see Fig. 2).

Carr and Simpson (1978) have reported on the responses of brachial DRG to wing bud extirpation. It is difficult to compare their results with ours since they present their data in the form of degeneration indices, that is, the percentage of degenerating cells in the total populations. Our results are in general agreement; however, there are differences in two minor points. $\Lambda$ ccording to Carr and Simpson (1978), the VL population of normal brachial DRG shows a peak of degeneration at $5^{1 / 2}$ days (the starting point of their investigation), and the degeneration index declines from then on. We found no degeneration up to stage 29 (51/2 to 6 days) and a distinct peak during days 6 and 7 , followed by a decline. Furthermore, the target-deprived VL population in their material undergoes a second peak of degeneration at $8 \frac{1}{2}$ days which is not apparent in our material.

Reduction of neuronal death by NGF. We have shown in a previous publication (Hamburger et al., 1981) that daily injections of $6 \mu \mathrm{g}$ of NGF, beginning at stage 21 (31/2 days), reduce naturally occurring neuronal death in brachial DRG very substantially in both populations (see NGFX controls in Figs. 1 and 2). However, in the VL population, a slight rise in the number of degenerating cells was observed toward the end of the degeneration period (Hamburger et al., 1981, Fig. 6). We assumed then that neurons which were rescued earlier died eventually. We shall come back to this point.

In the present experiment, in which the early wing bud extirpation had increased neuronal death drastically and had shifted the degeneration process in the VL population, the first two NGF injections at $3 \frac{1}{2}$ and 4 days have no effect on the initial phase of degeneration of VI. neurons. However, when degeneration reaches a peak in uninjected embryos at stage 27 (51/2 days), NGF treatment reduces the number of degenerating cells by $50 \%$. In the subsequent phase, during which cell death declines sharply both in injected and noninjected embryos, the effect of extraneous NGF supply becomes more pronounced. At stage 30 ( 7 days) all cells that would have died in the target-deprived DRG are rescued. The fact that NGF can make up for a deficiency of a trophic maintenance agent both in normal embryonic DRG and following early wing bud extirpation is significant. It gives strong support to the widely accepted notion that normally occurring and experimentally induced neuronal death are caused by a deficiency in the same agent and that both differ only in degree. Furthermore, our belief that NGF or a related molecule is actually produced by limb tissue is strengthened.

The case for the DM population is even more impressive. In normal development, the cell loss in this population is considerably greater than that of the VL population (compare Figs. 6 and 7 in Hamburger et al., 1981; see also wing extirpation controls in Figs. 1 and 2). The removal of the target doubles the number of degenerating neurons at the peak of the degeneration period (stage 35 , 9 days; see Fig. 2). The NGF injections result in the survival of all neurons which would have fallen victim to the removal of their targets (Fig. 2). We attribute the greater effectiveness of the NGF treatments in this case to the fact that the degeneration period of the DM population is much later than that of the VL population; hence, a greater amount of NGF has accumulated at the critical period.

A second degeneration period. We have reported above that one can recognize three phases of the degeneration curve of VL neurons following wing bud extirpation (WX in Fig. 1). In the first phase, a steep rise is followed immediately by a steep decline (stages 24 to 30,4 to 7 days). The second phase is a plateau (stages 30 to 33,7 to 8 days), followed in the third phase by a gradual decline (stages 33 to 36,8 to 10 days). The NGFX curve parallels the WX curve only during the first phase of massive degeneration. While a plateau is maintained in the wing extirpation curve, the curve for wing extirpation combined with NGF injections shows a second rise, and the two curves bisect at stage 33 ( $71 / 2$ to 8 days). From then on, cell death in the NGFX series remains consistently higher than in WX; this is an apparent contradic- 
tion to our claim concerning the role of $\mathrm{NGF}$ as a maintenance agent. In fact, at stage 36 , when neuronal death has subsided in the WX series, the level of cell death in the NGF-injected embryos is still high. In other words, most, if not all, neurons which have been saved during the early massive degeneration phase die during a second, extended degeneration period.

A similar phenomenon, although on a smaller scale, had been observed in the previous study of NGF effects on normally occurring death in the VL population (Hamburger et al., 1981, Figs. 4 and 6). We had explained this in terms of a dosage effect; namely, that the accumulated dose of NGF, at the daily rate of $6 \mu \mathrm{g}$, was not sufficient to maintain the life of the previously rescued neurons. We propose that, in principle, the same explanation holds in the present case. However, we find a discrepancy between the present data and previously published data (Hamburger et al., 1981) with respect to the quantitative aspects of the second degeneration period; this point requires a further comment. The left (control) DRG 15 in the series of wing extirpation combined with NGF injections (Fig. 1, open circles, dotted lines) shows a very high number of degenerating VL cells between stages 31 and 36 which, at its peak, reaches an average of 250 cells. In contrast, the previously obtained figures for secondary loss of VL cells in DRG 15 of NGF-injected embryos (Hamburger et al., 1981, Fig. 6, solid circles, dotted lines) amounted to only about 70 cells at the peak. However, the present and previously published data are in agreement on one important point. In both instances, the second degeneration period starts, peaks, and ends at the same stages. Hence, the discrepancy between the present and the previously obtained data is only a quantitative difference. We believe that we can account for it in terms of differences in NGF administration. In the previous series, the total amount of NGF which the embryos received was greater than in the present series. Perhaps more importantly, in the 1981 series, the injections were made into the yolk sac and in the present series into the amniotic cavity. It could be that the latter procedure is less effective. At any rate we believe that inadequate supply and retention of NGF, particularly in older stages, accounts for the secondary loss of VL cells in both sets of experiments. The fact that the injection of much higher doses of NGF at the second degeneration peak (stage 34) reduced the number of degenerating cells by half supports our argument.

The DM population does not show any significant amount of neuronal death, following daily injections of the standard dose of NGF, at least up to stage 38 (day 12), when our observation period ends (Fig. 2). Obviously, the requirements of the DM neurons for a maintenance agent are lower for these stages than those of the VL neurons. The fact that VL neurons are considerably larger than DM neurons, at least up to day 12 , may be related to this difference.

Concluding remarks. The question of whether NGF is the normally occurring trophic maintenance factor produced by the target structures of NGF-sensitive neurons is still unresolved. The investigation of Ebendal et al. (1980) indicates that NGF is actually produced in the rat iris in vivo. We have tried to obtain indirect evidence for the notion that NGF is the naturally occurring maintenance factor of DRG in the chick embryo. First, we have shown that NGF is transported retrogradely and selectively from target areas in the leg to lumbar DRG neurons during the critical stages when the survival of these neurons depends on an extraneous maintenance agent (Brunso-Bechtold and Hamburger, 1979). Second, we have demonstrated that daily injections of NGF from $4 \frac{1}{2}$ days on reduce normally occurring death in the VL population of thoracic DM populations by $60 \%$ and rescue practically all neurons in the thoracic DM population and in both populations of brachial DRG (Hamburger et al., 1981). Finally, in the present experiment, the capacity of NGF to keep alive embryonic sensory neurons which would otherwise die was subjected to a more severe test; the incidence of cell loss was increased substantially by target removal, that is, early wing bud extirpation. In this situation, daily injections of NGF reduced the very high mortality of the VL population substantially, although the supply of NGF was not sufficient to keep these neurons alive for a prolonged period. However, the dosage which we applied was sufficent to rescue the considerable number of DM neurons which would have been doomed by the wing extirpation. The fact that NGF provides an effective substitute for the normal maintenance factor for embryonic brachial DRG by preventing both normally occurring and experimentally induced neuron loss suggests that NGF is actually produced in the limb tissues. Furthermore, the experimental results reaffirm the notion that normally occurring and experimentally induced neuronal death have a common cause and differ only in degree.

\section{References}

Brunso-Bechtold, J.K., and V. Hamburger (1979) Retrograde transport of nerve growth factor in chicken embryo. Proc. Natl. Acad. Sci. U. S. A. 76: 1494-1496.

Carr, V. M. and S. B. Simpson (1978) Proliferative and degenerative events in the early development of chick dorsal root ganglia. J. Comp. Neurol. 182: 741-755.

Carr, V. M., and S. B. Simpson (1982) Rapid appearance of labelled degenerating cells in the dorsal root ganglia after exposure of chick embryos to tritiated thymidine. Dev. Brain. Res. 2: 157-162.

Cowan, W. M., and E. Wenger (1967) Cell loss in the trochlear nucleus of the chick during normal development and after radical extirpation of the optic vesicle. J. Exp. Zool. 164: 267280.

Ebendal, T., L. Olson, A. Seiger, and K. -O. Hedlund (1980) Nerve growth factor in the rat iris. Nature 286: 25-28.

Hamburger, V. (1934) The effects of wing bud extirpation on the development of the central nervous system in chick embryos. J. Exp. Zool. 68: 449-494.

Hamburger, V. (1958) Regression versus peripheral control of differentiation in motor hypoplasia. Am. J. Anat. 102: 365410.

Hamburger, V. (1975) Cell death in the development of the lateral motor column of the chick embryo. J. Comp. Neurol. 160: $535-546$.

Hamburger, V., and H. L. Hamilton (1951) A series of normal stages in the development of the chick embryo. J. Morphol. 88: 49-92.

Hamburger, V., and R. Levi-Montalcini (1949) Proliferation, differentiation and degeneration in the spinal ganglia of the 
chick embryo under normal and experimental conditions. J. Exp. Zool. 111: 457-502.

Hamburger, V., and R. Oppenheim (1982) Naturally occurring neuronal death in vertebrates. Neurosci. Comment. 1: 39-55.

Hamburger, V., J. K. Brunso-Bechtold, and J. W. Yip (1981) Neuronal death in the spinal ganglia of the chick embryo and its reduction by nerve growth factor. J. Neurosci. 1: 6071.

Harper, G.P., and H. Thoenen (1980) Nerve growth factor: biological significance, measurement and distribution. J. Neurochem. 34: 5-16.

Honig, M. (1982) The development of sensory projection patterns in embryonic chick hindlimb. J. Physiol. (Lond.) 330: $175-202$
Landmesser, L., and G. Pilar (1974) Synapse formation during embryogenesis on ganglion cells lacking a periphery. J. Physiol. (Lond.) 241: 715-736.

Levi-Montalcini, R., and P. N. Angeletti (1968) Nerve growth factor. Physiol. Rev. 48: 534-569.

Oppenheim, R. W., I. -W. Chu-Wang, and J. L. Maderdrut (1978) Cell death of motoneurons in the chick embryo spinal cord. III. The differentiation of motoneurons prior to their induced degeneration following limb-bud removal. J. Comp. Neurol. 177: 87-112.

Pannese, E. (1976) An electron microscopic study of cell degeneration in chick embryo spinal ganglia. Neuropathol. Appl. Neurobiol. 2: 247-267. 